\title{
Vacuum-formed retainers more effective than Hawley retainers
}

\author{
Abstracted from \\ Rowland $\mathrm{H}$, Hichens $\mathrm{L}$, Williams $\mathrm{A}$, et al. \\ The effectiveness of Hawley and vacuum-formed retainers: a single-center randomised controlled trial. \\ Am J Orthod Dentofacial Orthop 2007; 132: 730-737 \\ Address for correspondence: Jonathan Sandy, Bristol Dental Hospital, Lower Maudlin Street, \\ Bristol BS1 2LY, UK. E-mail: jonathan.sandy@bristol.ac.uk
}

\section{Question: In the first 6 months after active orthodontic treatment are vacuum-formed retainers more effective than Hawley retainers?}

Design A randomised controlled trial (RCT) was performed in a single orthodontic practice.

Intervention The aim was to compare the clinical effectiveness of Hawley and vacuum-formed retainers (VFR) over a 6-month period of retention. Three hundred and ninety-seven patients were treated by a specialist orthodontist and randomly allocated to wear either Hawley retainers ( $n=196)$ or VFR $(n=201)$.

Outcome measure A blinded examiner analysed the records of maxillary and mandibular dental casts at debond and at 6 months into retention, assessing tooth rotation mesial to the first permanent molar, intercanine and intermolar widths, and Little's Index of Irregularity. Results Three hundred and fifty-five individuals participated through to the conclusion of the trial, giving a completion rate of $89 \%$. The results showed significantly greater changes in irregularity of the incisors in the Hawley group than in the VFR group at 6 months. Conclusions VRF are more effective than Hawley retainers at holding the correction of the maxillary and mandibular labial segments.

\section{Commentary}

Retention is required after orthodontic treatment to minimise both relapse of the teeth towards their pretreatment position and facial growth-related changes. Although many practitioners will undoubtedly have their preferred retention regimes, there is a paucity of data to support which one is the most clinically effective. ${ }^{1}$

This study asks whether there is any difference in the clinical effectiveness of Hawley and VFR over the 6-month period following debonding. The method is a well-designed RCT, and some features really make this study stand out. The power calculation aims to detect a clinically meaningful contact point displacement difference of $0.2 \mathrm{~mm}$. The required sample size was therefore very large: 200 patients in each group. This was achieved by recruiting patients not from a hospital setting but from a single specialist orthodontic practice, which has the added benefit of making the results applicable to the very setting where most orthodontic treatment is carried out: primary care. It also makes it the largest published study to date investigating these retainers.

Trial participants were appropriately randomised and accounted for throughout the trial, as demonstrated in the CONSORT diagram. The outcome measures were Little's Index of Irregularity, tooth rotation, intercanine and intermolar widths, and overjet and overbite, with values compared at debond and 6 months. The Hawley group was instructed to wear their retainers fulltime for 3 months, then for $12 \mathrm{~h}$ each day. The VFR group was to wear the retainers fulltime for 1 week, then $12 \mathrm{~h}$ per day. Thus, as the authors acknowledge, the study does not directly compare two different retainers, but two retention regimes that are used in everyday clinical practice.

A statistically significant difference of $0.56 \mathrm{~mm}$ was found for incisor irregularity in the mandibular incisors. This difference could be considered clinically significant if the displacement is isolated to one tooth. This is interesting, as the Hawley design incorporated an acrylated labial bow (which ought to afford greater control of tooth position), and the VFR were only worn fulltime for 1 week.

The issues of cost effectiveness and patient satisfaction have been addressed in a parallel study, which also makes for interesting reading. If acceptability of one particular retainer influences cooperation, then longer-term followup may provide further useful information.

There is consensus that more high-quality studies are needed to answer important questions in clinical orthodontics. This collaborative research helps to answer one such question, and demonstrates what can be achieved by harnessing the advantages of primary and secondary care settings.

Practice point

- In the 6 months following debond, vacuum-formed retainers are more effective than Hawley retainers in maintaining labial segment alignment

Jan Ledvinka

Orthodontist, Sydney, Australia

1. Littlewood SJ, Millett DT, Doubleday B, Bearn DR, Worthington HV. Retention procedures for stabilising tooth position after treatment with orthodontic braces. Cochrane Database Syst Rev 2006; issue 1.

Evidence-Based Dentistry (2009) 10, 47. doi:10.1038/sj.ebd.6400650 